\title{
Prioritizing heart transplantation during the COVID-19 pandemic
}

\author{
Keki R. Balsara MD, MBA ${ }^{1}$ ( ) Z Zakiur Rahaman MD $^{1}$ | Emily Sandhaus BSN ${ }^{2}$ | \\ Jordan Hoffman MD ${ }^{1}$ | Sandip Zalawadiya MD ${ }^{2}$ | William McMaster MD ${ }^{1}$ | \\ Joann Lindenfeld $\mathrm{MD}^{2}$ | Mark Wigger $\mathrm{MD}^{2}$ | Tarek Absi $\mathrm{MD}^{1}$ | \\ Douglas M. Brinkley $\mathrm{MD}^{2}$ | Jonathan Menachem $\mathrm{MD}^{2}$ | Lynn Punnoose $\mathrm{MD}^{2}$ | \\ Suzie Sacks $\mathrm{MD}^{2}$ | Kelly Schlendorf MD, $\mathrm{MSCl}^{2}$ | Ashish S. Shah MD ${ }^{1}$
}

\footnotetext{
${ }^{1}$ Department of Cardiac Surgery, Vanderbilt University Medical Center, Nashville,

Tennessee, USA

${ }^{2}$ Division of Cardiology, Department of Medicine, Vanderbilt University Medical Center, Nashville, Tennessee, USA

\section{Correspondence}

Keki R. Balsara, MD, MBA, Vanderbilt University Medical Center, Nashville, TN 37232, USA.

Email: Keki.r.balsara@vumc.org
}

\begin{abstract}
Background: Coronavirus disease 2019 (COVID-19) has significantly impacted the healthcare landscape in the United States in a variety of ways including a nationwide reduction in operative volume. The impact of COVID-19 on the availability of donor organs and the impact on solid organ transplant remains unclear. We examine the impact of COVID-19 on a single, large-volume heart transplant program.

Methods: A retrospective chart review was performed examining all adult heart transplants performed at a single institution between March 2020 and June 2020. This was compared to the same time frame in 2019. We examined incidence of primary graft dysfunction, continuous renal replacement therapy (CRRT) and 30-day survival.
\end{abstract}

Results: From March to June 2020, 43 orthotopic heart transplants were performed compared to 31 performed during 2019. Donor and recipient demographics demonstrated no differences. There was no difference in 30-day survival. There was a statistically significant difference in incidence of postoperative CRRT (9/31 vs. 3/43; $p=.01)$. There was a statistically significant difference in race $(23 \mathrm{~W} / 8 \mathrm{~B} / 1 \mathrm{AA}$ vs. $30 \mathrm{~W} / 13 \mathrm{~B} ; p=.029)$.

Conclusion: We demonstrate that a single, large-volume transplant program was able to grow volume with little difference in donor variables and clinical outcomes following transplant. While multiple reasons are possible, most likely the reduction of volume at other programs allowed us to utilize organs to which we would not have previously had access. More significantly, our growth in volume was coupled with no instances of COVID-19 infection or transmission amongst patients or staff due to an aggressive testing and surveillance program.

KEYWORDS

COVID-19, heart transplantation, resource allocation 


\section{1 | INTRODUCTION}

Heart transplantation remains the definitive therapy for patients who suffer from end-stage heart failure. Since it was first performed in 1967, the number of patients who have benefited from this therapy has continued to increase. ${ }^{1,2}$ The past two decades have seen the introduction of new, improved immunosuppression which has allowed for longer allograft survival and innovations in donor recovery. The organ donor pool has been expanded by the utilization of hepatitis $C$ donors and ex vivo perfusion platforms. ${ }^{3}$ These developments hold promise for the meaningful increase in the number of adult heart transplants performed in the United States.

The spring of 2020 saw the widespread recognition of a novel coronavirus (coronavirus disease 2019 [COVID-19]) which has had a global impact. In the United States, the affect has been wide ranging. Nowhere has this been more apparent than the healthcare landscape. An increase in demand for hospital beds for COVID-19 positive patients coupled with hospital-driven initiatives to reduce non-emergent treatments has resulted in a nation-wide reduction in operative volume. ${ }^{4}$ Moreover, patient concerns regarding infection with COVID-19 has resulted in many patients deferring medical care for both major and minor concerns. ${ }^{5}$ At its worst, we witnessed reduction in case volume between $20 \%$ and $90 \%$ compared to last year across all service lines at our hospital.

The impact of COVID-19 on the availability of donor organs and the impact on solid organ transplant remains unclear. Here we examine the impact of COVID-19 on a single, large-volume heart transplant program.

\section{2 | METHODS}

Institutional Review Board approval at the Vanderbilt University Medical Center was obtained. We performed a retrospective chart review examining all adult heart transplants performed between March 2020 and June 2020. This was compared to the same time frame in 2019. We examined demographic data and outcomes which included incidence of primary graft dysfunction (PGD), continuous renal replacement therapy (CRRT), length of stay and 30-day survival. In addition, donor variables including hepatitis $C$ status and distance from our transplant center were examined. Statistical analysis was performed with Microsoft Excel.

\section{3 | RESULTS}

The first confirmed case of COVID-19 in the state of Tennessee was on March 5, 2020. By June 30, 2020, the total number of confirmed cases in the state was 43,806 . Over this same period of time, our total cardiac volume was 488 cases compare to 521 cases during March-June 2019. The most significant decrease in volume was seen in April 2020 where volume was reduced by nearly $30 \%$ compared to the previous year.
From March-June 2020, 43 total orthotopic heart transplants were performed. This includes three re-transplants and seven multiorgan transplants (6 heart/kidney; 1 heart/liver; 0 heart/lung). The average recipient age was $54.2 \pm 13.9$ years and 29 recipients were male and 14 were female. Thirty recipients were Caucasian. Thirteen recipients were African American. There were 11 blood type A recipients, seven blood type $B$ recipients, one blood type $A B$ recipient and 24 blood type $O$ recipients. Sixteen patients had preoperative durable left ventricular assist devices (LVAD). The average listing status at time of transplant was $3.3 \pm 1.4$. The average time on the wait list was $147.44 \pm 195.85$ days.

From March-June 2019, 31 total orthotopic heart transplants were performed. This includes three re-transplants and three multiorgan transplants ( 3 heart/kidney; 0 heart/liver; 0 heart/lung). The average recipient age was $47.5 \pm 13.3$ years and 18 recipients were male and 13 were female. Twenty-three patients were Caucasian. Eight patients were African American. One patient was Asian American. There were 15 blood type A recipients, five blood type $B$ recipients, one blood type $A B$ recipient and 10 blood type $O$ recipients. Eight patients had preoperative durable LVAD. The average listing status at time of transplant was $3.0 \pm 0.99$. The average time on the wait list was $112.94 \pm 260.11$ days. There was no significant difference in recipient demographic variables across groups except for race of the transplant recipients $(p=.029)$ (Table 1$)$.

Donor variables over these two time periods were not different (Table 2). From March-June 2020, the average donor age was $29.1 \pm 8.3$ years and 30 donors were male and 13 were female. Thirty-one donors were Caucasian. Seven donors were African American. One donor was Hispanic. One donor was Native American. Ten donors were hepatitis $C$ positive. Twenty donors were characterized as high risk. The average time of allograft travel to the recipient hospital was $88.49 \pm 53.5 \mathrm{~min}$.

From March to June 2019, the average donor age was $32.0 \pm 8.8$ years and 18 donors were male and 13 were female. Twenty-six

TABLE 1 Heart transplant recipient demographics for 2019 and 2020

\begin{tabular}{|lll|} 
& March-June 2019 & March-June 2020 \\
\hline Number of recipients & 31 & 43 \\
\hline Age (years) & $47.5+/-13.1$ & $54.2+/-13.9$ \\
\hline Gender & $18 \mathrm{M} / 13 \mathrm{~F}$ & $29 \mathrm{M} / 14 \mathrm{~F}$ \\
\hline Pre-op VAD & 8 & 16 \\
\hline Re-transplant & 3 & 3 \\
\hline Race & $23 \mathrm{C} / 8 \mathrm{AA} / 1 \mathrm{~A}$ & $30 \mathrm{C} / 13 \mathrm{AA}$ \\
\hline Blood type & $15 \mathrm{~A} / 5 \mathrm{~B} / 1 \mathrm{AB} / 10 \mathrm{O}$ & $11 \mathrm{~A} / 7 \mathrm{~B} / 1 \mathrm{AB} / 24 \mathrm{O}$ \\
\hline Status at listing & $3.0+/-.99$ & $3.3+/-1.4$ \\
\hline Time on waitlist (days) & $112.94+/-260.11$ & $147.44+/-195.85$ \\
\hline
\end{tabular}

Note: There was a statistically significant difference in race of recipients between the two groups $(p=.029)$. Gender: $M=$ male; $F=$ female. Race: $\mathrm{C}=$ Caucasian; $\mathrm{AA}=$ African American; $\mathrm{A}=$ Asian American. 
TABLE 2 Heart transplant donor demographics for 2019 and 2020

\begin{tabular}{lll} 
& March-June 2019 & March-June 2020 \\
\hline Age (years) & $32.0 \pm 8.8$ & $29.1 \pm 8.3$ \\
\hline Gender & $18 \mathrm{M} / 13 \mathrm{~F}$ & $30 \mathrm{M} / 13 \mathrm{~F}$ \\
\hline Race & $26 \mathrm{C} / 3 \mathrm{AA} / 2 \mathrm{H}$ & $31 \mathrm{C} / 7 \mathrm{AA} / 1 \mathrm{H} / 1 \mathrm{NA}$ \\
$\mathrm{HCV}+$ & 15 & 10 \\
\hline Travel time (min) & $87.81 \pm 36.05$ & $88.49 \pm 53.5$ \\
\hline
\end{tabular}

Note: There were no statistically significant differences. Gender: $\mathrm{M}=$ male; $\mathrm{F}=$ female. Race: $\mathrm{C}=$ Caucasian; $\mathrm{AA}=$ African American; $\mathrm{A}=$ Asian American; $\mathrm{H}=$ Hispanic; $\mathrm{NA}=$ Native American.

Abbreviation: $\mathrm{HCV}$, hepatitis $\mathrm{C}$ virus.

donors were Caucasian. Three donors were African American. Two donors were Hispanic. Fifteen donors were hepatitis $C$ positive. Seventeen donors were characterized as high risk. The average time of allograft travel to the recipient hospital was $87.87 \pm 36.05 \mathrm{~min}$. In addition, there was no difference in the number of local versus imported organs over these two periods of time.

Of the 43 transplants performed between March and June 2020, 41 achieved 30-day survival. Four recipients needed postoperative CRRT. The average total length of stay was $18.2 \pm 8.1$ days and the average intensive care unit length of stay was $6.1 \pm 3.1$ days. Four patients had PGD and six patients had rejection ( $2 \mathrm{R}$ or greater) at 30 days. $^{6}$

Of the 31 transplants performed between March and June 2019, 30 achieved 30-day survival. Nine patients needed postoperative CRRT. The average total length of stay was $23.0 \pm 13.4$ days and the average intensive care unit length of stay was $11.0 \pm 8.7$ days. Three patients had PGD and three patients had rejection ( $2 \mathrm{R}$ or greater) at 30 days. The only significant difference in recipient outcome was the incidence of CRRT ( $p=.01$ ) (Table 3).

There was no evidence of acute, postoperative infection of any recipient with COVID-19 during this time period.

\section{4 | DISCUSSION}

Heart transplantation remains the definitive therapy for patients with end stage heart disease. The number of heart transplants performed in the United States has gradually increased to approximately 3000/year over the past 20 years. $^{7}$ The recent past has seen the introduction of a variety of means by which the donor pool may increase including ex vivo support platforms, older donors and extended criteria donors.

However, much of the potential benefit of these advances have not been recognized in the past year due to a world-wide pandemic secondary to COVID-19. In the United States, more than nine-million individuals have been infected and nearly 250,000 individuals have died as a result of infection. ${ }^{8}$ The impact of this pandemic on the healthcare landscape has yet to be fully realized but it is likely to be
TABLE 3 Heart transplant outcomes for 2019 and 2020

\begin{tabular}{lll} 
& March-June 2019 & March-June 2020 \\
\hline 30 Day survival & 30 & 41 \\
\hline LOS (ICU) (days) & $23.0 \pm 13.4$ & $18.2 \pm 8.1$ \\
\hline LOS (total) (days) & $11.0 \pm 8.7$ & $6.1 \pm 3.1$ \\
\hline PGD & 3 & 4 \\
\hline Rejection at 30 days & 3 & 6 \\
\hline CRRT post-op & 9 & 4 \\
\hline
\end{tabular}

Note: There was a statistically significant difference in the incidence of continuous renal replacement therapy (CRRT) between the two groups $(p=.01)$. Rejection at 30 days was defined as $2 \mathrm{R}$ or greater at biopsy. Abbreviations: ICU, intensive care unit; LOS, length of stay; PGD, primary graft dysfunction.

substantial. The late spring and early summer saw widespread mitigation attempts including the shuttering of schools, places of work and businesses. Initial uncertainty regarding the scale of infection, the need for healthcare resources and the availability of personal protective equipment prompted many centers to institute moratoriums on all non-emergent care. ${ }^{9-11}$ Anecdotal evidence suggests that many individuals did not seek medical care for non-COVID related chronic illness and more still did not present for urgent/emergent care. In fact, a recent study examined 717,103 patients in the Society of Thoracic Surgeons Database who underwent cardiac surgery between January 2017 and June 2020. They observed a 53\% reduction in the average monthly cardiac surgical volume with the onset of the pandemic including a $65 \%$ decrease in elective and $40 \%$ reduction in nonelective cases. Geographically, the hardest hit areas were the mid-Atlantic states with a $71 \%$ reduction in volume and the New England states with a $63 \%$ reduction. The Southeast, where our center is located, experienced a nearly $50 \%$ reduction in volume over this same period. There was also a $110 \%$ increase in the observed to expected operative mortality nationwide. ${ }^{12}$

The impact of COVID-19 on solid organ transplant, particularly orthotopic heart transplant, remains unclear. Scientific Registry of Transplant Recipients data suggests that many programs in geographically hard-hit areas significantly reduced or stopped all solid organ transplants. ${ }^{13}$ Solid organ allocation continued to be driven by the 11 regions in the United States and no changes in organ allocation occurred. Decisions regarding listing and transplantation were at the discretion of individual programs. Transplant volume in the United States over the period of this study does appear to be impacted by geography. Large-volume programs in the southeastern and western portions of the United States maintained volumes, on average, of 6-10 transplants/month. Programs in the northeast performed, on average, six transplants/ month with a handful of programs performing no transplants in the months of May and June. The driver behind these observations has yet to be fully elucidated. As one of the largest heart transplant programs in the country, we were able to navigate the pandemic while keeping our program open. 
At the height of the COVID lockdown between March and June 2020 , we were able to increase our transplant volume by nearly $40 \%$. Our ability to do this is attributable to both nation-wide trends regarding transplant during this time as well as institutional initiatives which prioritized the maintenance of programs which offered lifesaving therapies. An examination of our program's performance during this time highlights many of the tensions that predated COVID-19 for transplant programs nationwide and perhaps provides a model for how to expand orthotopic heart transplant availability in the post-COIVD era.

As we look at demographic information for donors and recipients, a few interesting observations were made. There was no significant difference in age of donor or recipient, no significant difference in status at the time of transplant and no significant difference in travel time of the allograft to our center. There was, however, a statistically significant difference in race of recipients between the two time periods, with the COVID era having a greater number of recipients of color. The specific reason for this remains unclear. Many studies demonstrate that people of color have a reduced incidence of transplant and have more complications compared to Caucasian recipients. ${ }^{14}$ Access to care, comorbidities and financial limitations have all been identified as potential reasons. Our program has made a concerted effort to identify potentially reversable barriers to transplant and address them in a multidisciplinary fashion such that certain socioeconomic limitations do not prevent patients from transplant. It is a testament to the success of these endeavors that during a pandemic which disproportionately affected people of color, these patients' access to life-saving heart transplant was not impacted.

Outcomes including 30-day survival, length of stay, PGD and rejection were unchanged. We made no changes to perioperative or immunosuppressant management during this period. There was a significant decrease in the incidence of postoperative renal replacement therapy in those patients who underwent transplant during the COVID era. This, most likely, had less to do with COVID and more to do with some changes to our intra- and postoperative management of these patients. We have seen an overall trend towards less utilization of renal replacement therapy in our posttransplant patients.

More significant, perhaps, is the focus our institution placed on maintaining and even expanding access to transplant during this time. We speculate that while many programs curtailed transplant activity due to capacity constraints secondary to COVID hospitalization and personal protective equipment conservation efforts, our institution elected to prioritize the maintenance of our transplant programs. The end result, potentially, was that we had access to organs that, under normal circumstances, would have been utilized by surrounding programs.

From an institutional perspective, we implemented a rigorous program of testing, monitoring and isolating pretransplant and posttransplant patients from the remainder of patients in the hospital. This included testing on admission and immediately before transplant, colocalizing transplant patients in a single intensive care unit and a single step-down unit and the most rigorous utilization of personal protective equipment in all interactions with transplant patients. In addition, listed and posttransplant patients were asked to isolate themselves from all but their immediate caregivers. The end result was that at no point during this study period was a patient or staff member taking care of these patients noted to develop COVID-19. In addition, travel restrictions and limited access to donor hospitals forced us to rethink procurement strategies. We relied much more heavily on local surgeons to recover organs for us, a shift from traditional practice. These initiatives could very well represent a paradigm shift in the manner in which organs are procured, eliminating certain personnel constraints that are common in a program of our size.

An additional struggle identified were the natural concerns expressed by listed patients regarding the risk of COVID-19 infection in the hospital, the implications of COVID-19 infection in recently transplanted patients and projecting the time frame over which COVID-19 might impact their individual chances of receiving organs. Much effort was invested in being fully transparent in the initiatives we were implementing to augment patient safety. The most compelling argument was our acknowledgment that the duration of COVID-19 was uncertain and that its impact on organ availability was unknown. With this knowledge, most patients elected to undergo transplant, recognizing that it was unclear when an additional opportunity might present itself.

As an institution, we were not immune to the impact of COVID-19. Like many institutions across the country, we scaled back "elective" procedures to ensure enough resources for urgent/emergent care. This naturally raised a number of questions surrounding which services would be offered and what would be considered urgent/emergent. The same ethical principles inherent to transplant practices continue to apply during the COVID-19 era, but the balance between autonomy, beneficence, nonmaleficence, and justice will be inherently fluid. Transplantation is inherently a resource intensive service. Recipients demand close monitoring by staff, long stays in an intensive care unit, utilization of ventilators and blood products, and use of personal protective equipment. Additionally, immunosuppressed individuals are at higher risk of being susceptible to a highly infectious, aerosolized virus. Moreover, staff safety concerns regarding travel on planes and prolonged periods of time in the operating room or ward must be considered. Perhaps most importantly is the acknowledgment that transplantation is never an elective procedure. Patients awaiting heart transplant often have no alternative therapy that can provide long-lasting results. While inotropic therapy and ventricular assist device support are options for some, they do not provide the durable results found with transplant. Without knowing when this pandemic will end, is it fair to deprive patients of potentially more life-extending therapy? Another consideration is the fact that donor organs are not widely available. For some patients who have waited months or years on a waitlist, should they be deprived of transplant when a suitable donor is identified? While there are no clear answers to these ethical considerations, we sought to find a delicate balance between access to care for the 
many as a result of the pandemic and specialized care for the few in need of a life-saving transplant.

In the final analysis, COVID-19 has dramatically affected all aspects of our lives. Perhaps nowhere has this been more readily apparent than in the healthcare space. In spite of that impact, we demonstrate that orthotopic heart transplants can continue to be performed safely. While we cannot deny that we potentially benefited from other programs' reduction in volume, what we have truly demonstrated is that a rigorous testing and surveillance program allows one to continue providing this life-saving therapy, while ensuring the health and safety of both patients and providers.

\section{CONFLICT OF INTERESTS}

The authors declare that there are no conflict of interests.

\section{ORCID}

Keki R. Balsara (D) https://orcid.org/0000-0002-3502-5705

\section{REFERENCES}

1. Barnard CN. The operation: a human cardiac transplant: an interim report of a successful operation performed at Groote Schuur, Hospital, Cape Town. S Afr Med J; 1967;41(48):1271-1274.

2. Holm AM, Mehra MR, Courtwright A, et al. Ethical considerations regarding heart and lung transplantation and mechanical circulatory support during the COVID-19 pandemic: an ISHLT COVID-19 Task Force statement. J Heart Lung Transplant. 2020;39(7):619-626.

3. Schlendorf $\mathrm{KH}$, Zalawadiya S, Shah AS, et al. Expanding heart transplant in the era of direct-acting antiviral therapy for hepatitis C. JAMA Cardiology. 2020;8(2):87-99.

4. Emanuel EJ, Persad G, Upshur R, et al. Fair allocation of scarce medical resources in the time of COVID-19. New England Journal of Medicine; 2020; 382:2049-2055.

5. American College of Surgeons. American Society of Anesthesiologists, Association of perioperative Registered Nurses, American Hospital Association. Joint statement: roadmap for resuming elective surgery after COVID 19 pandemic. 2020.
6. Jeesup M, Drazner MH, Book W, et al. ACC/AGA/HFSA/ISHLT/ACP advanced training statement on advanced heart failure and transplant cardiology (revision of the ACCF/AHA/ACP/HFSA/ISHLT 2010 clinical competence statement on management of patients with advanced hear failure and cardiac transplant): a report of the ACC competency management committee. Circulation: Heart Failure. 2017;10(6).

7. Goff RR, Uccellini K, Lindblad K, et al. A change of heart: preliminary results of the US 2018 adult heart allocation revision. Am J Transplant (AJT). 2020;20(10):2781-2790.

8. World Health Organization. Coronavirus disease (COVID-19) pandemic. 2020. https://www.who.int/emergencies/diseases/novelcoronavirus-2019

9. White $\mathrm{DB}$, Lo B. A framework for rationing of ventilators and critical care beds during COVID-19 pandemic. JAMA. 2020;323(18): 1773-1174.

10. Galvan NTN, Moreno NF, Garza JE, et al. Donor and transplant candidate selection for solid organ transplantation during the COVID-19 pandemic. Am J Transplant (AJT). 2020;20(11): 3113-3122.

11. Ritschl PV, Nevermann N, Wiering L, et al. Solid organ transplantation programs facing lack of empiric evidence in the COVID-19 pandemic: a by-proxy society recommendation consensus approach. Am J Transplant (AJT). 2020;20(7):1826-1836.

12. Ngyuen TC. The effect of COVID-19 on adult cardiac surgery in the United States: Analysis of the Society of Thoracic Surgeons adult cardiac surgery database. Presented at STS. 2021.

13. Scientific Registry of Transplant Recipients. COVID-19 changes to January 2022 PSRs/OSRs. https://www.srtr.org

14. Cox K, Carter K, Lirette $\mathrm{S}$, et al. Donor and recipient racial mismatch impacts thoracic organ transplant survival. J Heart Lung Transplant. 2019;38(4):S266-S267.

How to cite this article: Balsara KR, Rahaman Z, Sandhaus E, et al. Prioritizing heart transplantation during the COVID-19 pandemic. J Card Surg. 2021;36:3217-3221.

https://doi.org/10.1111/jocs.15731 